\title{
Clinical Benefits of Lymph Node Dissection in Intrahepatic Cholangiocarcinoma: A Retrospective Single-institution Study
}

\author{
TATSUNORI MIYATA, YO-ICHI YAMASHITA, TAKANOBU YAMAO, NAOKI UMEZAKI, MASAYO TSUKAMOTO, \\ YUKI KITANO, KENSUKE YAMAMURA, KOTA ARIMA, TAKAYOSHI KAIDA, SHIGEKI NAKAGAWA, \\ KATSUNORI IMAI, DAISUKE HASHIMOTO, AKIRA CHIKAMOTO, TAKATOSHI ISHIKO and HIDEO BABA \\ Department of Gastroenterological Surgery, Graduate School of Life Sciences, \\ Kumamoto University, Kumamoto, Japan
}

\begin{abstract}
Background/Aim: The prognostic effect of lymph node dissection (LND) in intrahepatic cholangiocarcinoma (ICC) remains unclear. The aim of this study was to reveal the clinical benefits of LND in patients with ICC after curative hepatic resections. Patients and Methods: We retrospectively analyzed 64 patients with ICC who underwent primary curative resections between June 2002 and February 2016 and evaluated the survival benefits of $L N D$. Results: We performed LND on patients suspected to have LN metastasis before operation (23/64 cases; $35.9 \%)$. There was no significant difference in prognosis between the $L N D(+)$ group $(n=22)$ and the $L N D(-)$ group $(n=41)$; relapse-free survival (RFS): $p=0.635$ and overall survival $(O S): p=0.347$. Among the groups, $p N O(n=17), p N 1(n=7)$ and $p N X$ (without $L N D$ or $L N$ sampling; $n=39$ ), the survival curves of pNO and pNX groups were similar $(p=0.568)$. Conclusion: Special attention should be given to the indication of $L N D$ and the practice of secure operation.
\end{abstract}

Intrahepatic cholangiocarcinoma (ICC) is a primary liver cancer with a typically poor prognosis (1). According to the Japanese registry compiled by the Liver Cancer Study Group of Japan, which has 18,213 newly-registered patients, approximately $94 \%$ of patients had hepatocellular carcinoma (HCC) and only $4.1 \%$ had ICC (2), while a subsequent report revealed an increase in the incidence of ICC (3). ICC is, thus, considered a highly malignant neoplasm because it is frequently associated with lymph node (LN) involvement,

Correspondence to: Yo-ichi Yamashita, MD, Ph.D., F.A.C.S., Department of Gastroenterological Surgery, Graduate School of Life Sciences, Kumamoto University, 1-1-1 Honjo, Chuo-ku, Kumamoto 860-0811, Japan. Tel: +81 963735211, Fax: +81 963714378, e-mail: y-yama@kumamoto-u.ac.jp

Key Words: Intrahepatic cholangiocarcinoma, lymph node dissection, prognosis. intrahepatic metastasis and peritoneal dissemination (4). Although surgery has been considered a potentially curative treatment for ICC, high recurrence rates and poor adjuvant therapies have resulted in a 5-year survival for resectable ICC of $48 \%$, with median survival ranging from 30 to 43 months $(5,6)$.

Although many local and regional treatment modalities are available for patients with ICC, most modalities, including local ablation and hepatic intra-arterial therapies, have marginal therapeutic roles as a result of inherent limitations and/or lack of a durable tumor response (7). Surgical resection has only been shown to improve long-term survival for patients with ICC at present (8).

In clinicopathological factors, LN metastasis is a very strong poor prognostic factor $(9,10)$; however, the indications and roles of lymph node dissection (LND) in patients with ICC are still subject to discussion. Although some consider that the standard surgical procedure for ICC is a hepatectomy combined with extensive LND, not all centers support routine LND (11). In addition, there are not clear guidelines on LND, including the standard route and extent of LND. Some reports have shown LND for ICC is not effective in patients' prognosis with $\operatorname{ICC}(4,12)$. However, other reports have shown that regional LND should be considered a standard part of surgical therapy for patients undergoing resection of ICC (13). Therefore, the aim of the current study was to evaluate the clinical benefits of LND in patients with ICC after curative resections.

\section{Patients and Methods}

Patients. We performed a retrospective analysis of consecutive patients to examine the surgical outcomes of patients with ICC who underwent curative resections. Between June 2002 and February 2016, 78 patients underwent laparotomy for ICC at Kumamoto University Hospital, Kumamoto, Japan. Of these 78 patients, 64 underwent primary curative resections, which resulted in pathological curative resection (pR0). Among 14 excluded patients, 10 patients were surgeries for recurrence and 4 were non-curative 
Table I. Clinicopathological features in 64 patients with ICC.

\begin{tabular}{|c|c|c|c|}
\hline \multirow[t]{2}{*}{ Variables } & \multicolumn{2}{|c|}{ LND } & \multirow[t]{2}{*}{$p$-Value } \\
\hline & $\mathrm{N}=41(-)$ & $\mathrm{N}=23(+)$ & \\
\hline \multicolumn{4}{|l|}{ Background characteristics } \\
\hline Gender & & & 1.000 \\
\hline Male & 10 & 6 & \\
\hline Female & 31 & 17 & \\
\hline Age & $66.4 \pm 1.6$ & $65.1 \pm 2.2$ & 0.618 \\
\hline Hepatitis B infection: yes & 5 & 2 & 0.662 \\
\hline Hepatitis C infection: yes & 10 & 3 & 0.266 \\
\hline ICGR15 $(\%)$ & $10.1 \pm 0.9$ & $9.2 \pm 1.1$ & 0.511 \\
\hline CEA (ng/ml) & $44.1 \pm 33.1$ & $3.7 \pm 44.2$ & 0.467 \\
\hline CA19-9 (U/ml) & $938 \pm 726$ & $403 \pm 970$ & 0.660 \\
\hline \multicolumn{4}{|l|}{ Tumor-related and pathological factors } \\
\hline Gross type & & & 0.530 \\
\hline Mass-forming & 35 & 17 & \\
\hline Periductal infiltrating & 3 & 3 & \\
\hline Mass-forming+ Periductal infiltrating & 3 & 3 & \\
\hline Tumor size $(\mathrm{mm})$ & $40.8 \pm 3.7$ & $39.4 \pm 4.9$ & 0.828 \\
\hline Tumor number & $1.6 \pm 0.3$ & $1.3 \pm 0.4$ & 0.509 \\
\hline Vascular invasion: yes/no & $16 / 25$ & $12 / 11$ & 0.431 \\
\hline pN status $(0 / 1 / \mathrm{X})$ & $0 / 2 / 39$ & $17 / 6 / 0$ & $<0.0001$ \\
\hline pStage (I/II/III/IV) & $1 / 15 / 17 / 8$ & $1 / 6 / 9 / 7$ & 0.294 \\
\hline \multicolumn{4}{|l|}{ Preoperative outcomes } \\
\hline Operating time (min) & $399 \pm 14$ & $520 \pm 18$ & $<0.0001$ \\
\hline Blood loss (ml) & $552 \pm 88$ & $765 \pm 116$ & 0.147 \\
\hline Transfusion: yes & 6 & 5 & 0.504 \\
\hline Bile duct resection: yes & 0 & 5 & 0.004 \\
\hline Postoperative complication $(\mathrm{CD} \geq \mathrm{III})$ : yes & 4 & 8 & 0.022 \\
\hline Mortality: yes & 0 & 1 & 1.000 \\
\hline
\end{tabular}

CC, Intrahepatic cholangiocarcinoma; LND, lymph node dissection; ICG R15, indocyanine green retentIion rate at 15 minutes; CEA, carcinoembryonic antigen; CA19-9, carbohydrate antigen 19-9; CD, Clavien-Dindo classification.

resections. Therefore, we included the 64 patients with primary curative resections and analyzed the clinical benefits of LND. Mortality case was excluded when we analyzed survival rate. The median follow-up time for censored patients was 37 months. We performed 5-fluorouracil-based adjuvant chemotherapy to the patients with lymph node metastasis or vascular invasion if patients' performance status was 0 or 1 . Written informed consent was obtained from each patient. The Institutional Review Board approved this study.

Indications for lymph node dissection. We performed LND in patients who were suspected to have $\mathrm{LN}$ metastasis in preoperative computed tomography (CT) scan or positron emission tomography (PET) or macroscopic findings during the operations (14). We suspected LN metastasis if LN enlargement was detected in CT scan or fluorodeoxyglucose accumulated at LN in PET. In such cases, we mainly resected $\mathrm{LN}$ along the common hepatic artery (\#8 LN) and $\mathrm{LN}$ in the hepatoduodenal ligament (\#12 LN) and, occasionally, added on some LN, such as LN on the right cardiac (\#1 LN), LN

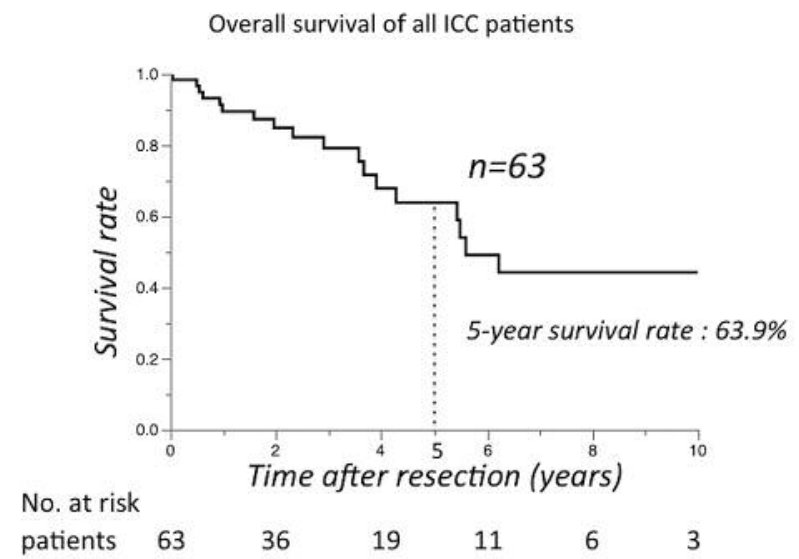

Figure 1. Overall survival of all 63 patients with intrahepatic cholangiocarcinoma (ICC) who underwent curative resections. The 5year-survival rate was $63.9 \%$.

along the lesser curvature (\#3 LN), LN along the left gastric artery (\#7 LN) and LN on the posterior surface of the pancreatic head (\#13 LN). Sampling of LN was not included in LND.

Statistical analysis. Comparison between the two groups was examined by the Student's $t$-tests for continuous variables and $\chi^{2}$ tests or Fisher's exact tests for nominal variables. The continuous variables in Table I show mean \pm standard deviation (SD). The overall survival rate (OS) and relapse-free survival rate (RFS) were calculated using the Kaplan-Meier method from the date of surgery to date of last follow-up. The comparisons of survival curves were performed using the log-rank test. Significance levels were set at $p<0.05$. All tests were two-sided. All statistical analyses were performed using JMP software (Version 12; SAS Institute, Cary, NC, USA).

\section{Results}

Clinicopathological characteristics and perioperative outcomes. Table I shows the clinicopathological characteristics and perioperative outcomes of 64 patients with pathologically-confirmed ICC who were included in the study. Twenty-three patients (35.9\%) underwent LND. In pathological nodal (pN) status, $39(60.9 \%)$ patients who did not have LND or who had LN sampling were described as $\mathrm{pNX}(\mathrm{n}=39,60.9 \%)$. There were no differences in background characteristics between the two groups, such as gender, age and tumor marker. In tumor-related and pathological factors, $\mathrm{pN}$ status was significantly different $(p<0.0001)$. In the LND (+) group, the positive rate of LN metastasis was $26.1 \%$. On the other hand, in the LND (-) group, two patients $(3.1 \%)$ were positive for $\mathrm{LN}$ metastasis. These two patients underwent LN sampling without frozen pathology and diagnosed as positive for LN metastasis in hematoxylin \& eosin staining after the operations. In perioperative data, operating time was significantly longer in 


\section{Relapse-free survival and overall survival of patients with ICC according to LND}

A

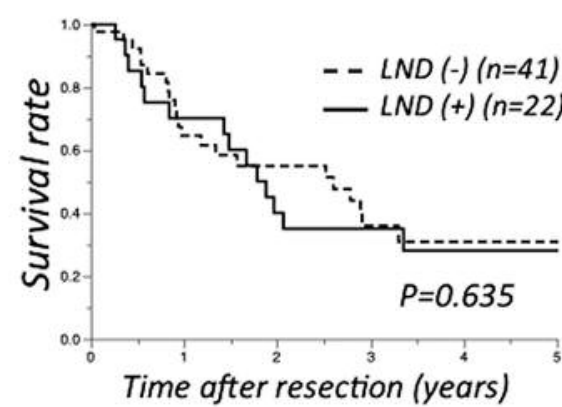

No. at risk

\begin{tabular}{|c|c|c|c|c|c|c|}
\hline Years & 0 & 1 & 2 & 3 & 4 & 5 \\
\hline LND (-) & 41 & 24 & 16 & 9 & 7 & 5 \\
\hline LND (+) & 22 & 15 & 9 & 7 & 5 & 5 \\
\hline
\end{tabular}

B Overall survival

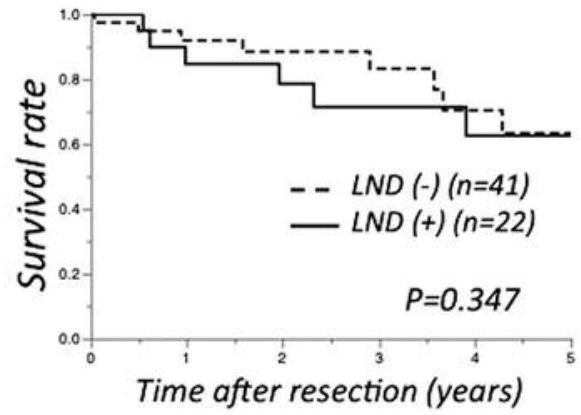

No. at risk

\begin{tabular}{|c|c|c|c|c|c|c|}
\hline Years & 0 & 1 & 2 & 3 & 4 & 5 \\
\hline LND (-) & 41 & 33 & 23 & 17 & 12 & 9 \\
\hline LND (+) & 22 & 17 & 14 & 11 & 8 & 8 \\
\hline
\end{tabular}

Figure 2. (A) Relapse-free survival (RFS) and (B) overall survival (OS) in patients with intrahepatic cholangiocarcinoma (ICC) who underwent curative resections with or without lymph node dissection (LND). There is no significant difference between the two groups in RFS ( $p=0.635)$ and OS ( $p=0.347)$, respectively.

the $\mathrm{LND}(+)$ group $(p<0.0001)$. Five patients were subjected to bile duct resections in the $\operatorname{LND}(+)$ group. The complication rate, which was defined as Clavien-Dindo classification III or more, was significantly higher in the LND (+) group $(p=0.022)(15)$.

The survival benefits of LND. Figure 1 shows the overall survival curve of all patients in current study, with 1-, 3- and 5-year-survival rates being $89.5 \%, 79.2 \%$ and $63.9 \%$, respectively. Figure 2 demonstrates no significant differences on OS and RFS between the $\operatorname{LND}(+)$ and the $\operatorname{LND}(-)$ groups (RFS: $p=0.635$, OS: $p=0.347$ ). RFS at 5 years was $28.1 \%$ in the $\mathrm{LND}(+)$ group and $30.9 \%$ in the $\mathrm{LND}(-)$ group. OS at 5 years was $62.6 \%$ in the $\mathrm{LND}(+)$ group and $63.4 \%$ in the LND(-) group. Although pN1 group had significantly poor prognosis than $\mathrm{pN} 0$ and $\mathrm{pNX}$ group $(p=0.035), \mathrm{pN} 0$ and $\mathrm{pNX}$ groups had similar prognoses $(p=0.568)$ (Figure 3$)$. The clinical courses of 8 patients in the $\mathrm{pN} 1$ group are summarized in Table II. Two patients $(25 \%)$ had a long-term survival without recurrence after hepatectomies with LND.

\section{Discussion}

In the present study, we report our experience with ICC as single-institution study and demonstrate that LND may not improve RFS and OS in 63 patients with ICC after curative

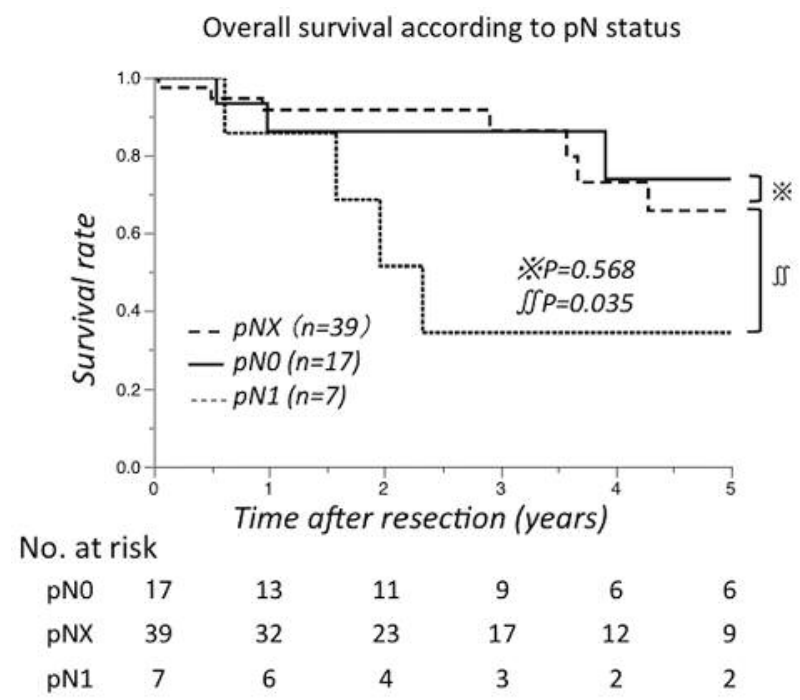

Figure 3. Overall survival in each of $p N$ stage ( $p N O, p N 1$ and $p N X)$ after curative resections. Patients with pN1 had significantly poor prognosis than $p N O$ and $p N X$ patients $(p=0.035)$.

hepatic resections. We also report that the patients who were diagnosed as pNX or pNO had similar prognosis.

LN metastases have been reported as a poor prognostic factor for patients with ICC (10). Weber et al. discussed the 
Table II. Patients' characteristics, perioperative and prognosis data with LN metastasis.

\begin{tabular}{|c|c|c|c|c|c|c|c|c|}
\hline Gender & Age & LND & Surgical procedure & Location of LN metastasis & Site of recurrence & RFS (years) & Dead or Alive & OS (years) \\
\hline M & 71 & $(+)$ & Left hemi hepatectomy & 12 & - & 8.34 & Alive & 8.34 \\
\hline $\mathrm{F}$ & 58 & $(+)$ & Extended left hepatectomy & 12 & Bone & 0.40 & Dead & 0.61 \\
\hline $\mathrm{F}$ & 74 & $(+)$ & Left lateral segmentectomy & 1 & Lung, LN & 1.96 & Dead & 1.96 \\
\hline $\mathrm{F}$ & 68 & $(+)$ & Extended left hepatectomy & 12 & Liver, bone & 1.48 & Dead & 2.32 \\
\hline M & 78 & $(+)$ & Right hepatectomy & 12 & - & 0.22 & Dead & 0.22 \\
\hline $\mathrm{F}$ & 51 & $(+)$ & Extended left hepatectomy & 8,12 & - & 3.03 & Alive & 3.03 \\
\hline $\mathrm{F}$ & 79 & $(-)$ & S5 segmentectomy & 13 & Liver, lung, bone & 0.61 & Dead & 1.58 \\
\hline $\mathrm{F}$ & 73 & $(-)$ & Extended right hepatectomy & 12 & Liver & 0.35 & Alive & 0.69 \\
\hline
\end{tabular}

LND, Lymph node dissection; LN, lymph node; RFS, relapse-free survival; OS, overall survival; M, male; F, female.

role of LND concluding that LND should be considered a standard part of surgical therapy for patients undergoing resection of ICC (13). On the other hand, Shimada et al. concluded that LND in ICC was not suitable to improve patients' survival (4). Moreover, Morine et al. showed that prophylactic systematic LND did not affect surgical outcomes in ICC without LN metastasis (16). In the present study, LND did not tend to prolong RFS and OS. We also showed that patients, who did not undergo LND and those without LN metastasis who did, had similar survival outcomes ( $p=0.568)$ (Figure 3 ), thus suggesting that LND may not improve the survival in patients without $\mathrm{LN}$ metastasis. In addition, recurrence rate was statistically similar between the three groups ( $\mathrm{pN} 0 ; 58.8 \%, \mathrm{pN} 1 ; 71.4 \%$, pNX; $43.6 \%, p=0.288)$. On the other hand, two patients who underwent LND and had LN metastasis had a good prognosis after hepatectomies. Therefore, hepatectomies with LND may be effective and approved for patients who were suspected to have LN metastasis.

However, we have some concerns about avoiding LND. First, the difficulty of diagnosis of LN metastasis has to be taken into account. Adachi et al. mentioned the difficulties of diagnosis by CT or PET and reported that inflammation, such as cholangitis or necrosis of the original tumor, causes LN enlargement (14). Second, the diagnosis of LN metastasis is affected by the location of LN sampling. It is important to note which LNs are being resected. In the present study, positive LN status was seen in five patients (62.5\%) with \#12 LN metastasis, a patient (12.5\%) with \#13 $\mathrm{LN}$ metastasis, a patient with \#8 $\mathrm{LN}$ and \#12 LN metastasis, as well as a patient $(12.5 \%)$ with \#1 LN metastasis (Table II). In these eight patients, two patients $(25 \%)$ did not undergo LND. Six of eight patients had \#12 LN metastasis. Li et al. showed that the LN in hepatoduodenal ligament was the most common LN metastasis site in 104 patients with ICC (17). Therefore, these results suggested that the routine LN sampling of \#12 LN was useful to detect whether LN metastasis was present or not.
We also demonstrated that patients with LND had longer operating time, more blood loss and more postoperative complications. Basically, postoperative complications are one of poor prognostic factors in cancer therapy $(18,19)$. Some reports show that postoperative complications can lead to an immune modulation, a decrease in tumor surveillance and, possibly, an increased risk of disease recurrence and diseasespecific death $(20,21)$. Therefore, we should pay attention as to perform hepatectomies without postoperative complications in order to improve patients' prognosis by avoidance of routine LND.

This study has several limitations. First, this study was performed as a retrospective single-institution study with small number of patients. Second, the policy of LN sampling and LND was not consistent; for example, \#1 and \#3 LN in left-sided ICC and \#8 LN in right-sided ICC $(22,4,12)$. Therefore, some larger and prospective study with a consistent policy for LND is necessary to reveal the influence of LND in ICC on clinical benefits.

In conclusion, we should pay attention to the indication of LND in patients with ICC and perform safe operation without postoperative complications to improve patient's prognosis.

\section{Conflicts of Interest}

The Authors declare that they have no conflict of interest and this study was not funded.

\section{References}

1 Aljiffry M, Abdulelah A, Walsh M, Peltekian K, Alwayn I and Molinari M: Evidence-based approach to cholangiocarcinoma: A systematic review of the current literature. J Am Coll Surg 208: 134-147, 2009.

2 Ikai I, Arii S, Okazaki M, Okita K, Omata M, Kojiro M, Takayasu K, Nakanuma Y, Makuuchi M, Matsuyama Y, Monden M and Kudo M: Report of the 17th Nationwide Follow-up Survey of Primary Liver Cancer in Japan. Hepatol Res 37: 676691, 2007. 
3 Endo I, Gonen M, Yopp AC, Dalal KM, Zhou Q, Klimstra D, D'Angelica M, DeMatteo RP, Fong Y, Schwartz L, Kemeny N, O'Reilly E, Abou-Alfa GK, Shimada H, Blumgart LH and Jarnagin WR: Intrahepatic cholangiocarcinoma: Rising frequency, improved survival, and determinants of outcome after resection. Ann Surg 248: 84-96, 2008.

4 Shimada M, Yamashita Y, Aishima S, Shirabe K, Takenaka K and Sugimachi K: Value of lymph node dissection during resection of intrahepatic cholangiocarcinoma. Br J Surg 88: 1463-1466, 2001.

5 Spolverato G, Kim Y, Ejaz A, Alexandrescu S, Marques H, Aldrighetti L, Gamblin TC, Pulitano C, Bauer TW, Shen F, Sandroussi C, Poultsides G, Maithel SK and Pawlik TM: Conditional probability of long-term survival after liver resection for intrahepatic cholangiocarcinoma: A multi-institutional analysis of 535 patients. JAMA Surg 150: 538-545, 2015.

6 Spolverato G, Yakoob MY, Kim Y, Alexandrescu S, Marques HP, Lamelas J, Aldrighetti L, Gamblin TC, Maithel SK, Pulitano C, Bauer TW, Shen F, Poultsides GA, Marsh JW and Pawlik TM: Impact of complications on long-term survival after resection of intrahepatic cholangiocarcinoma. Cancer 121: 2730-2739, 2015.

7 Dodson RM, Weiss MJ, Cosgrove D, Herman JM, Kamel I, Anders R, Geschwind JF and Pawlik TM: Intrahepatic cholangiocarcinoma: Management options and emerging therapies. J Am Coll Surg 217: 736-750.e734, 2013.

8 Nathan H, Pawlik TM, Wolfgang CL, Choti MA, Cameron JL and Schulick RD: Trends in survival after surgery for cholangiocarcinoma: A 30-year population-based SEER database analysis. J Gastrointest Surg 11: 1488-1497, 2007.

9 Horino K, Beppu T, Komori H, Masuda T, Hayashi H, Okabe H, Takamori $\mathrm{H}$ and Baba $\mathrm{H}$ : Evaluation of mass-forming intrahepatic cholangiocarcinoma with viral hepatitis. Hepatogastroenterology 59: 1217-1219, 2012.

10 Nakagawa T, Kamiyama T, Kurauchi N, Matsushita M, Nakanishi K, Kamachi H, Kudo T and Todo S: Number of lymph node metastases is a significant prognostic factor in intrahepatic cholangiocarcinoma. World J Surg 29: 728-733, 2005.

11 Patel T: Increasing incidence and mortality of primary intrahepatic cholangiocarcinoma in the United States. Hepatology 33: 1353-1357, 2001.

12 Yamashita Y, Taketomi A, Morita K, Fukuhara T, Ueda S, Sanefuji K, Iguchi T, Kayashima H, Sugimachi K and Maehara Y: The impact of surgical treatment and poor prognostic factors for patients with intrahepatic cholangiocarcinoma: Retrospective analysis of 60 patients. Anticancer Res 28: 2353-2359, 2008.

13 Weber SM, Ribero D, O'Reilly EM, Kokudo N, Miyazaki M and Pawlik TM: Intrahepatic cholangiocarcinoma: expert consensus statement. HPB (Oxford) 17: 669-680, 2015.
14 Adachi T, Eguchi S, Beppu T, Ueno S, Shiraishi M, Okuda K, Yamashita Y, Kondo K, Nanashima A, Ohta M, Takami Y, Noritomi T, Kitahara K and Fujioka H: Prognostic impact of preoperative lymph node enlargement in intrahepatic cholangiocarcinoma: A multi-institutional study by the Kyushu Study Group of liver surgery. Ann Surg Oncol 22: 2269-2278, 2015.

15 Dindo D, Demartines $\mathrm{N}$ and Clavien PA: Classification of surgical complications: A new proposal with evaluation in a cohort of 6336 patients and results of a survey. Ann Surg 240: 205-213, 2004.

16 Morine Y and Shimada M: The value of systematic lymph node dissection for intrahepatic cholangiocarcinoma from the viewpoint of liver lymphatics. J Gastroenterol 50: 913-927, 2015.

17 Li DY, Zhang HB, Yang N, Quan Y and Yang GS: Routine lymph node dissection may be not suitable for all intrahepatic cholangiocarcinoma patients: Results of a monocentric series. World J Gastroenterol 19: 9084-9091, 2013.

18 Doussot A, Lim C, Gomez Gavara C, Fuks D, Farges O, Regimbeau JM and Azoulay D: Multicentre study of the impact of morbidity on long-term survival following hepatectomy for intrahepatic cholangiocarcinoma. Br J Surg 103: 1887-1894, 2016.

19 Miyata T, Yamashita YI, Yamao T, Umezaki N, Tsukamoto M, Kitano Y, Yamamura K, Arima K, Kaida T, Nakagawa S, Imai K, Hashimoto D, Chikamoto A, Ishiko T and Baba H: Prognostic impacts of postoperative complications in patients with intrahepatic cholangiocarcinoma after curative operations. Int J Clin Oncol, 2017. doi: 10.1007/s10147-017-1099-9. [Epub ahead of print]

20 Balkwill $\mathrm{F}$ and Mantovani A: Inflammation and cancer: Back to Virchow? Lancet 357: 539-545, 2001.

21 Lundy $\mathbf{J}$ and Ford CM: Surgery, trauma and immune suppression. Evolving the mechanism. Ann Surg 197: 434-438, 1983.

22 Aishima S, Kuroda Y, Nishihara Y, Iguchi T, Taguchi K, Taketomi A, Maehara $\mathrm{Y}$ and Tsuneyoshi M: Proposal of progression model for intrahepatic cholangiocarcinoma: Clinicopathologic differences between hilar type and peripheral type. Am J Surg Pathol 31: 1059-1067, 2007.

Received March 10, 2017

Revised March 19, 2017

Accepted March 20, 2017 